\title{
Recovery of the potential from $I$-function
}

\author{
A.G. Ramm \\ Department of Mathematics \\ Kansas State University, Manhattan, KS 66506-2602, USA \\ ramm@math.ksu.edu
}

\begin{abstract}
The inverse scattering problem on half-axis, in other words, in the spherically symmetric case, consists of finding the unknown potential from a suitable class from the scattering data $\mathcal{S}$. The other problem of practical interest is to find this potential from the spectral data $d \rho(\lambda)$. In the literature there are recovery procedures for finding the potential from the spectral or from the scattering data. Define the $I$-function: $I(k):=\frac{f^{\prime}(0, k)}{f(k)}$, where $f(x, k)$ is the Jost solution. Constructive ways to find $I(k)$ from $d \rho(\lambda)$ and vice versa, and $I(k)$ from $\mathcal{S}$ and vice versa are given. The theory of Riemann problem is used as an essential tool. If $I(k)$ is found, then our methods allow one to construct the scattering data and from these recover the potential by the known procedure. Alternatively, one can construct from $I(k)$ the spectral data and from these find the potential by the known procedure.
\end{abstract}

MSC: 34A55

Keywords: I-function; Riemann problem; scattering data; spectral data; inverse problem.

\section{Introduction}

The operator $\ell u=-u^{\prime \prime}+q(x) u$ is defined in $H=L^{2}\left(\mathbb{R}_{+}\right), \mathbb{R}_{+}=[0, \infty)$ by the boundary condition $u(0)=0$. The $q$ is a real-valued function in $L_{1,1}:=\left\{q: \int_{0}^{\infty}(1+x)|q(x)| d x<\infty\right\}$. The spectral function

$$
\left\{\begin{array}{l}
d \rho(\lambda)=\frac{\lambda^{1 / 2} d \lambda}{\pi|f(\sqrt{\lambda})|^{2}}, \quad \lambda \geq 0, \\
\sum_{j=1}^{J} c_{j} \delta\left(\lambda+k_{j}^{2}\right) d \lambda, \quad \lambda<0 .
\end{array}\right.
$$


Here $\delta$ is the delta function, $f(k):=f(0, k), k:=\sqrt{\lambda}$, the function $f(x, k)$, the Jost solution, is defined as the unique solution to the problem

$$
\ell f-k^{2} f=0, \quad f=e^{i k x}+o(1), \quad x \rightarrow \infty ; \quad k>0 .
$$

The $c_{j}>0$ are norming constants (see [2] or [3])

$$
c_{j}=-2 i k_{j} \frac{f^{\prime}\left(0, i k_{j}\right)}{\dot{f}\left(i k_{j}\right)},
$$

where $f^{\prime}(0, k)=\left.\frac{\partial f(x, k)}{\partial x}\right|_{x=0}$ and $k_{j}>0$ are numbers such that

$$
f\left(i k_{j}\right)=0, \quad 1 \leq j \leq J .
$$

The numbers $i k_{j}$ are simple zeros of $f(k)$ in the upper half of the complex plane $\mathbb{C}$.

The $I$-function is defined as

$$
I(k):=\frac{f^{\prime}(0, k)}{f(k)} .
$$

Properties of $I$-function were studied in [3], where, among other things, it was proved that $I$-function is identical with the Weyl-Titchmarsh function.

The scattering data $\mathcal{S}$ is the collection:

$$
\mathcal{S}=\left\{S(k):=\frac{f(-k)}{f(k)}, k \geq 0, k_{j}, s_{j}, 1 \leq j \leq J\right\},
$$

where $k_{j}$ are defined in (1.4) and $s_{j}>0$ are the norming constants

$$
s_{j}=-2 i k_{j} \frac{1}{\dot{f}\left(i k_{j}\right) f^{\prime}\left(0, i k_{j}\right)}=\frac{c_{j}}{\left[f^{\prime}\left(0, i k_{j}\right)\right]^{2}},
$$

and $J$ is the number of negative eigenvalues of the Dirichlet operator $\ell$. This number is finite under our assumptions on $q$, (see, for example, [2]). By $\phi(x, k)$ let us denote the unique solution of the problem

$$
\ell \phi-k^{2} \phi=0, \quad \phi(0, k)=0, \quad \phi^{\prime}(0, k)=1 .
$$

It is known [2] that $d \rho(\lambda)$ and $\mathcal{S}$ each determines $q$ uniquely.

The results of this paper can be described as follows:

It is proved that $\{I(k)\}$, known for all $k \geq 0$, determines $d \rho(\lambda)$ uniquely and vice versa; that the scattering data $\mathcal{S}$ determines $\{I(k)\}$ uniquely and vice versa; explicit formulas are given for recovery processes. 
A consequence of the above results is the unique determination of the potential $q \in L_{1,1}$ from $\{I(k)\}$.

These results are obtained using the theory of the Riemann problem and other tools from complex analysis. The emphasis is on the explicit formulas for the recovery processes.

Let us formulate some of the results.

Theorem 1.1. The $I(k)$ determines $d \rho(\lambda)$ and $\mathcal{S}$ uniquely. Each of the data $d \rho(\lambda)$ or $\mathcal{S}$ determines $I(k)$ uniquely.

Remark. The author [4] published in 1987 paper [4] with the same title. There the problem of recovery of $q$ from $I$-function was posed and it was proved that $I$-function determines uniquely the potential. In the current paper much more is proved by the novel method based on the theory of Riemann problem. Namely, we prove now that both the spectral and the scattering data can be uniquely and explicitly calculated if the $I$-function is known and conversely, the $I$-function can be uniquely and explicitly calculated from the spectral or from the scattering data.

If $I(k)$ is given, then by the constructive methods, developed in this paper, one can construct the scattering data or/and the spectral data. From the spectral data one can uniquely recover the unknown potential using the known recovery procedure (see [2] or [3]). Also, from $I(k)$ one can construct the spectral data, and from these one can uniquely recover the unknown potential using the known method (see [2] or [3]).

\section{Proofs}

\subsection{Proof of the relation $I(k) \Rightarrow d \rho$.}

The zeros of $f(k)$ in $\mathbb{C}_{+}:=\{z: z \in \mathbb{C}, \operatorname{Im} z>0\}$ are simple poles of $I(k)$ because $\dot{f}\left(i k_{j}\right) \neq 0$ and $f^{\prime}\left(0, i k_{j}\right) \neq 0$ (see [3] and formula (1.3)). Thus, $I(k)$ determines uniquely the numbers $i k_{j}$ and, consequently, the numbers $k_{j}, 1 \leq j \leq J$. Define the numbers

$$
a_{j}:=\operatorname{Res}_{k=i k_{j}} I(k)=\frac{f^{\prime}\left(0, i k_{j}\right)}{\dot{f}\left(i k_{j}\right)} .
$$

Then, by formula (1.3), one has

$$
c_{j}=-2 i k_{j} a_{j}
$$


Note that $f(-k)=\overline{f(k)}$ and $f(0,-k)=\overline{f^{\prime}(0, k)}$ for real $k$, where the overline stands for complex conjugate. Thus,

$\operatorname{ImI}(k):=\frac{1}{2 i}\left(\frac{f^{\prime}(0, k)}{f(k)}-\frac{f^{\prime}(0,-k)}{f(-k)}\right)=\frac{f^{\prime}(0, k) f(-k)-f^{\prime}(0,-k) f(k)}{2 i|f(k)|^{2}}=\frac{k}{|f(k)|^{2}}$.

Here the known Wronskian formula was used

$$
f^{\prime}(0, k) f(-k)-f^{\prime}(0,-k) f(k)=2 i k .
$$

Thus, we have proved that $I(k)$ determines $d \rho(\lambda)$ uniquely and explicitly.

\subsection{Proof of the relation $d \rho(\lambda) \Rightarrow I(k)$.}

A trivial way to prove the relation $d \rho(\lambda) \Rightarrow I(k)$ is to recover $q(x)$ from $d \rho(\lambda)$ using the known Gel'fand-Levitan (GL) method, then construct the Jost function $f(x, k)$ uniquely as the solution to (1.2), then find $f(k)$ and $f^{\prime}(0, k)$, and then find $I(k)$ by formula $(1.5)$.

However, we want to show that $f(k)$ and $f^{\prime}(0, k)$ can be recovered uniquely and explicitly directly from $d \rho(\lambda)$, without finding $q$ from $d \rho(\lambda)$.

To recover $f(k)$ we solve the following Riemann problem

$$
f(k)=G(k) \frac{1}{f(-k)}, \quad G(k)=|f(k)|^{2}=f(k) f(-k) .
$$

If $d \rho(\lambda)$ is given, then $G(k)$ is known explicitly, see formula (1.1). The function $f(k)$ is analytic in $\mathbb{C}_{+}$, the function $\frac{1}{f(-k)}$ has finitely many simple poles in $\mathbb{C}_{-}:=\mathbb{C} \backslash \mathbb{C}_{+}$at the points $-i k_{j}, 1 \leq j \leq J$. Define the index of $G(k)$ as follows:

$$
i n d G(k):=\frac{1}{2 \pi} \Delta_{\mathbb{R}} \arg G(k)=\frac{1}{2 \pi i} \int_{-\infty}^{\infty} d \ln G(k)=\frac{1}{2 \pi i} \int_{-\infty}^{\infty} \frac{G^{\prime}(k)}{G) k} d k,
$$

where $\Delta_{\mathbb{R}} \arg G(k)$ is the increment of the argument of $G(k)$ when $k$ runs from $-\infty$ to $+\infty$ along the real axis.

Clearly,

$$
\operatorname{ind}(f(k) f(-k))=0 \text {. }
$$

Let us define

$$
\Phi_{-}(k):=\frac{w(-k)}{f(-k)}, \quad w(k):=\prod_{j=1}^{J} \frac{k-i k_{j}}{k+i k_{j}}
$$




$$
\Phi_{+}(k):=\frac{f(k)}{w(k)} .
$$

Then $\Phi_{+} \in H\left(\mathbb{C}_{+}\right)$, where $H\left(\mathbb{C}_{+}\right)$is the set of analytic in $\mathbb{C}_{+}$functions, $\Phi_{-} \in H\left(\mathbb{C}_{-}\right)$, and $(2.4)$ can be written as

$$
\Phi_{+}(k)=\frac{G(k)}{w(k) w(-k)} \Phi_{-}(k)=G(k) \Phi_{-}(k),
$$

because

$$
w(-k)=\frac{1}{w(k)} .
$$

Let us first assume that $f(0) \neq 0$. Since $i n d G(k)=0$, it follows from $(2.9)$ that

$$
\Phi_{+}(z)=\exp \frac{1}{2 \pi i} \int_{-\infty}^{\infty} \frac{\ln G(s)}{s-z} d s, \quad z \in \mathbb{C}_{+},
$$

see [1]. Therefore, by formula (2.8), one gets:

$$
f(k)=w(k) \exp \frac{1}{2 \pi i} \int_{-\infty}^{\infty} \frac{\ln G(s)}{s-k} d s, \quad \operatorname{Im} k>0 .
$$

On the real axis one has

$$
f(k)=w(k) \exp \left(\frac{1}{2 \pi i} \int_{-\infty}^{\infty} \frac{\ln G(s)}{s-k} d s+\frac{1}{2} \ln G(k)\right), \quad k \in \mathbb{R},
$$

where the known Sokhotski-Plemelj formula was used (see [1]):

$$
\frac{1}{2 \pi i} \int_{-\infty}^{\infty} \frac{g(t)}{t-k-i 0} d t=\frac{1}{2 \pi i} \int_{-\infty}^{\infty} \frac{g(t)}{t-k} d t+\frac{1}{2} g(k)
$$

and the integral on the right is understood as the Cauchy principal value.

Let us now assume that $f(0)=0$. Then $w(k)$ should be replaced by $w_{0}(k):=w(k) \frac{k}{k+i \kappa}$, where $\kappa>0$ is a number which is not equal to any of $k_{j}, 1 \leq j \leq J$, and $G(k)$ is replaced by $G_{0}(k):=G(k) \frac{k^{2}+\kappa^{2}}{k^{2}}$. Note that

$$
i n d G_{0}(k)=i n d G(k)+i n d\left(1+\frac{\kappa^{2}}{k^{2}}\right)=0+0=0,
$$

so formula (2.12) and (2.13) remain valid after replacing of $G$ by $G_{0}$.

Let us prove that $d \rho(\lambda)$ determines $f^{\prime}(0, k)$ uniquely. If not, then there are two different functions $f_{1}^{\prime}(0, k)$ and $f_{2}^{\prime}(0, k)$ each of which satisfies relation (2.3) with the same $f(k)$. 
Let

$$
h(k):=f_{1}^{\prime}(0, k)-f_{2}^{\prime}(0, k) .
$$

Then, subtracting from (2.3) with $f^{\prime}=f_{1}^{\prime}$ equation (2.3) with $f^{\prime}=f_{2}^{\prime}$, one gets

$$
h(k) f(-k)=h(-k) f(k) \quad \text { or } \quad \frac{h(k)}{f(k)}=\frac{h(-k)}{f(-k)} .
$$

The function $\frac{h(k)}{f(k)}$ is meromorphic in $\mathbb{C}_{+}$, and $\frac{h(-k)}{f(-k)}$ is meromorphic in $\mathbb{C}_{-}$. The only poles of $\frac{h(k)}{f(k)}$ in $\mathbb{C}_{+}$can be the simple poles at the points $i k_{j}$. However,

$$
f_{1}^{\prime}\left(0, i k_{j}\right)-f_{2}^{\prime}\left(0, i k_{j}\right)=0
$$

because the numbers $c_{j}$, see (1.3), and, therefore, the numbers $f^{\prime}\left(0, i k_{j}\right)$ are uniquely determined by $d \rho(\lambda)$ (recall that $f(k)$ is already determined and the numbers $\dot{f}\left(i k_{j}\right)$ are therefore known).

The relation $(2.16)$ and the differentiability of $h(k)$ prove that $\frac{h(k)}{f(k)}$ is analytic in $\mathbb{C}_{+}$and $\frac{h(-k)}{f(-k)}$ is analytic in $\mathbb{C}_{-}$. They agree on the real line and, therefore, they are analytic continuation of each other on the whole complex plane $\mathbb{C}$. The function $\frac{h(k)}{f(k)}$ is analytic in $\mathbb{C}$ and tends to zero at infinity, as we prove below. Consequently, $\frac{h(k)}{f(k)}=0$, so $h(k)=0$, and $f_{1}^{\prime}(0, k)=f_{2}^{\prime}(0, k)$. Thus, $f^{\prime}(0, k)$ is uniquely determined by $d \rho(\lambda)$.

Let us prove that $\frac{h(k)}{f(k)} \rightarrow 0$ as $|k| \rightarrow \infty$. Since

$$
f(x, k)=e^{i k x}+\int_{x}^{\infty} A(x, t) e^{i k t} d t
$$

one has

$$
\begin{aligned}
& f(k)=1+\int_{0}^{\infty} A(0, t) e^{i k t} d t:=1+a(k), \\
& f^{\prime}(0, k)=i k-A(0,0)+\int_{0}^{\infty} A_{x}(0, t) e^{i k t} d t:=i k-A_{0}+b(k) .
\end{aligned}
$$

Integrate by parts and rewrite (2.19) as

$$
f(k)=1-\frac{A_{0}}{i k}-\frac{1}{i k} \int_{0}^{\infty} A_{t}(0, t) e^{i k t} d t
$$

and get

$$
A_{0}=-\lim _{k \rightarrow \infty}[i k(f(k)-1)]
$$


This constant is known since $f(k)$ is known. Here we have used the relation

$$
\lim _{k \rightarrow \infty} \int_{0}^{\infty} A_{t}(0, t) e^{i k t} d t=0
$$

which follows from the Riemann-Lebesgue lemma because $A_{t}(0, t) \in L^{1}\left(\mathbb{R}_{+}\right)$. The last inclusion follows from the assumption $\int_{0}^{\infty}(1+s) \mid q(s) d s<\infty$ and from the known estimates (see, for example, [3]):

$$
|A(x, t)| \leq c \int_{\frac{x+t}{2}}^{\infty}|q(s)| d s, \quad\left|A_{t}(0, t)+\frac{1}{4} q(t / 2)\right| \leq c \int_{\frac{t}{2}}^{\infty}|q(s)| d s,
$$

where $c>0$ is a constant. Thus, formula (2.20) implies

$$
h(k)=f_{1}^{\prime}(0, k)-f_{2}^{\prime}(0, k)=b_{1}(k)-b_{2}(k) .
$$

It follows from (2.21) that $f(k) \rightarrow 1$ as $|k| \rightarrow \infty, \operatorname{Im} k \geq 0$. It follows from the definition (2.20) of $b(k)$ and from estimates $(2.23)$ that $b(k) \rightarrow 0$ as $|k| \rightarrow \infty, \operatorname{Im} k \geq 0$. Consequently,

$$
\lim _{|k| \rightarrow \infty, I m k \geq 0} \frac{h(k)}{f(k)}=0, \quad \lim _{|k| \rightarrow \infty, \operatorname{Im} k \leq 0} \frac{h(-k)}{f(-k)}=0 .
$$

From this and from formula (2.16) it follows that

$$
\lim _{|k| \rightarrow \infty} \frac{h(k)}{f(k)}=0 .
$$

Thus, $h=0$ and the proof of the uniqueness of recovery of $I(k)$ from $d \rho(\lambda)$ is completed.

We have recovered $f(k)$ explicitly from $d \rho(\lambda)$ and proved that $f^{\prime}(0, k)$ is uniquely determined by $d \rho(\lambda)$.

Let us prove that one can also recover $f^{\prime}(0, k)$ explicitly from $d \rho(\lambda)$. From (2.3), one gets

$$
\frac{f^{\prime}(0, k)}{f(k)}=\frac{f^{\prime}(0,-k)}{f(-k)}+\frac{2 i k}{|f(k)|^{2}}, \quad-\infty<k<\infty .
$$

We consider this problem as the problem of finding piecewise-analytic function from its jump across the contour $(-\infty, \infty)$. Compared with the standard problem of this type the difficulties are:

a) The function $\frac{f^{\prime}(0, k)}{f(k)}$ is not analytic in $\mathbb{C}_{+}$, and 
b) The function $\frac{2 i k}{|f(k)|^{2}}$ grows as $k \rightarrow \infty$.

Let us show how to overcome these difficulties.

Using formula (2.20), write (2.26) as

$$
\frac{b(k)}{f(k)}=\frac{b(-k)}{f(-k)}+\frac{2 i k-i k[f(-k)+f(-k)]}{|f(k)|^{2}}=\frac{b(-k)}{f(-k)}-\frac{i k[a(k)+a(-k)]}{|f(k)|^{2}}
$$

The function $\frac{b(k)}{f(k)}$ is meromorphic in $\mathbb{C}_{+}$with the only poles at the points $i k_{j}$, and these poles are simple. The residues $\frac{b\left(i k_{j}\right)}{\dot{f}\left(i k_{j}\right)}:=b_{j}, 1 \leq j \leq J$, are known since $f^{\prime}\left(0, i k_{j}\right)$ are known if $d \rho(\lambda)$ is known, see formula (1.3). Therefore, the function

$$
\psi_{+}(k):=\frac{b(k)}{f(k)}-\sum_{j=1}^{J} \frac{b_{j}}{k-i k_{j}}
$$

is analytic in $\mathbb{C}_{+}$and vanishes at infinity in $\mathbb{C}_{+}$.

The function

$$
\psi_{-}(k):=\frac{b(-k)}{f(-k)}+\sum_{j=1}^{J} \frac{b_{j}}{k+i k_{j}}
$$

is analytic in $\mathbb{C}_{-}$and vanishes at infinity in $\mathbb{C}_{-}$.

Equation (2.27) can be written as

$$
\psi_{+}(k)=\psi_{-}(k)-\frac{i k[a(k)+a(-k)]}{|f(k)|^{2}}-\sum_{j=1}^{J} \frac{b_{j}}{k-i k_{j}}-\sum_{j=1}^{J} \frac{b_{j}}{k+i k_{j}} .
$$

By the jump formula for the Cauchy integral one gets

$$
\psi_{+}(z)=\frac{1}{2 \pi i} \int_{-\infty}^{\infty} \frac{-\frac{i s[a(s)+a(-s)]}{|f(s)|^{2}}-\sum_{j=1}^{J} \frac{2 s b_{j}}{s^{2}+k_{j}^{2}}}{s-z} d s, \quad \operatorname{Im} z>0 .
$$

Thus

$$
b(k)=f(k)\left(\psi_{+}(k)+\sum_{j=1}^{J} \frac{b_{j}}{k-i k_{j}}\right), \quad f^{\prime}(0, k)=i k-A_{0}+b(k) .
$$

The integral in (2.31) converges because by formula (2.21) one has

$$
-i s[a(s)+a(-s)]=\int_{0}^{\infty} A_{t}(0, t)\left(e^{i s t}-e^{-i s t}\right) d t,
$$

where $A_{t}(0, t) \in L^{1}\left(\mathbb{R}_{+}\right)$by the estimate (2.23). Thus, $b(k)$ and, consequently, $f^{\prime}(0, k)$ are recovered explicitly.

This completes the recovery of $I(k)$ from $d \rho(\lambda)$. 


\subsection{Proof of the relation $I(k) \Rightarrow \mathcal{S}$}

As in section 2.1, $I(k)$ determines $k_{j}, 1 \leq j \leq J$, and $s_{j}$ by formula (1.7). Therefore, the data $\mathcal{S}$ are uniquely determined by $I(k)$ provided that $f(k)$ is found. In section 2.2 the function $f(k)$ was uniquely and explicitly recovered from $\operatorname{Im} I(k)$. Thus, $\mathcal{S}$ is found uniquely and explicitly from $I(k)$.

\subsection{Proof of the relation $\mathcal{S} \Rightarrow I(k)$}

A trivial way to find $I(k)$ from $\mathcal{S}$ is to use the known (Marchenko) procedure for finding $q$ from $\mathcal{S}$, then find $f(x, k)$ from from $q$, and then find $I(k)$.

Let us show that one can find $f(k)$ from $\mathcal{S}$ directly and explicitly using the Riemann problem, and then find $f^{\prime}(0, k)$ explicitly as was done in section

2.2. If $\mathcal{S}$ is known then $S(k)=\frac{f(-k)}{f(k)}$ is known and $k_{j}, 1 \leq j \leq J$, are known since $i k_{j}$ are simple poles of $S(k)$ in $\mathbb{C}_{+}$.

One has

$$
f(k)=S(-k) f(-k),
$$

since $S(-k)=S^{-1}(k)$ for $k \in \mathbb{R}$ because $S(-k)=\frac{f(k)}{f(-k)}=S^{-1}(k)$. Let

$$
\psi_{+}:=\frac{f(k)}{w(k)}, \quad \psi_{-}:=\frac{f(-k)}{w(-k)},
$$

and let $w(k)$ be defined in equation (2.7). Then (2.34) implies

$$
\psi_{+}(k)=\frac{S(-k) w(-k)}{w(k)} \psi_{-}(k)
$$

if $\operatorname{ind} S(-k)=2 J$ (that is, $f(0) \neq 0)$.

Clearly,

$$
i n d \frac{S(-k) w(-k)}{w(k)}=0,
$$

because

$$
\operatorname{ind} w(k)=J, \quad \text { ind } w(-k)=-J .
$$

It follows from (2.36) and (2.37) that

$$
\psi_{+}(k)=\exp \frac{1}{2 \pi i} \int_{-\infty}^{\infty} \ln \left(\frac{S(-t) w(-t)}{w(t)}\right) \frac{d t}{t-k}, \quad \text { Imk } \geq 0 .
$$

Therefore, $f(k)$ is found explicitly:

$$
f(k)=w(k) \psi_{+}(k), \quad \operatorname{Im} k \geq 0,
$$


where the integral in (2.39) is calculated by formula (2.14) for real $k$. Thus, $f(k)$ is recovered uniquely and explicitly from the scattering data $\mathcal{S}$. The $f^{\prime}(0, k)$ is recovered uniquely and explicitly as in Section 2.2. Therefore, $I(k)$ is recovered from $\mathcal{S}$.

If $f(0)=0$ then ind $S(-k)=2 J+1$, and in the above proof one replaces $w(k)$ by $w_{0}(k)$.

Theorem 1.1 is proved.

\section{References}

[1] Gahov, F., Boundary value problems, Pergamon Press, New York, 1966.

[2] Levitan, B., Inverse Sturm-Liouville problems, VNU Press, Utrecht, the Netherlands, 1987.

[3] Ramm, A. G., Inverse problems, Springer, New York, 2005.

[4] Ramm, A. G., Recovery of the potential from I-function, Math. reports of the Acad. Sci. Canada, 9, (1987), 177-182. 